\title{
Totgesagte leben länger: Stabilität und Kontinuität der Sozialpartnerschaft in Österreich.
}

\author{
Tobias Hinterseer ${ }^{\star}$
}

\section{Zusammenfassung}

Nach einer Reihe von Zäsuren des politischen Systems im Allgemeinen und der Sozialpartnerschaft im Besonderen, vor allem aber nach der Bildung einer Mitte-rechts-Regierung durch die Österreichische Volkspartei (ÖVP) und die Freiheitliche Partei Österreichs (FPÖ) im Jahr 20oo, konstatierte die österreichische Politikwissenschaft einen Verlust von politischer Einflussnahme beziehungsweise einen Rückgang der Gestaltungspotenziale der Sozialpartnerschaft und des Austrokorporatismus. Doch bereits während dieser "Zäsurzeit" und in weiterer Folge mit der Neuauflage der Großen Koalition kam es zu einem Wiederaufschwung der Sozialpartnerschaft, in der diese ihren Einfluss und ihre Machtposition wieder stärken und festigen konnte. Dieses Papier möchte die folgende Fragestellung beantworten: Was sind die Erklärungen für die Stabilität und Kontinuität der Sozialpartnerschaft in der Phase der Zurückdrängung zwischen den Jahren 2000 und 2006? Anhand der Analyse von zwei Gesetzgebungsprozessen wird gezeigt, dass institutionelle und akteursbezogene Faktoren und vor allem durch tradierte sozialpartnerschaftliche Identitäten geprägte Erwartungssicherheiten auch in Zeiten der politischen Zäsur und potenzieller politischer Entmachtung ein starker Garant für den Weiterbestand dieses Systems und die Kontinuität und Stabilität des Austrokorporatismus sind.

Schlagwörter: Sozialpartnerschaft, „ultrastability”, Korporatismus, Neo-Institutionalismus, akteurszentrierter Institutionalismus

\section{"There's life in the old dog yet" - The stability and continuity of the Austrian social partnership.}

Abstract

At the beginning of the millennium, scientists predicted a decline of the Austrian social partnership's political importance. However, during the time of repression the social partnership rediscovered its ability to affect recovery and regained political influence. Currently, the social partnership has demonstrated itself again to be wellgrounded in the labor law legislative process. Therefore this article approaches following question: How can the stability and continuity of the social partnership during the phase of repression (between the years 2000 and 2006) be explained? To answer this question two law-making processes during this period will be analyzed: it will be shown that institutional and actor-centered explanations as well as social partnership specific identities are the key explanations for the current continuity and stability of the Austro-Corporatism.

Keywords: Austrian social partnership, ultrastability, corporatism, neo-institutionalism, actor-centredinstitutionalism

\footnotetext{
* Tobias Hinterseer, Kammer für Arbeiter und Angestellte Salzburg. E-Mail: tobias.hinterseer@ak-salzburg.at
} 


\section{Einleitung: Die Sozialpartnerschaft zwischen Bedeutungslosigkeit und Wiederaufschwung: Die Zeit zwischen 2000 und 2006}

Das in Österreich etablierte System der industriellen Beziehungen stellt „(...) nicht den geläufigen interessenspolitischen Modus in kapitalistischen Gesellschaften (...)“ (Tálos 2008) dar. Globalisierungsprozesse, die europäische Integration wie auch internationale ökonomische Interdependenzen trugen Herausforderungen an die nationalen politischen und ökonomischen Handlungsspielräume der Sozialpartnerschaft heran (Dachs et. al 2006, Gerlich 1992, Karlhofer/Tálos 2006, Tálos 2005, 2001; Tálos/Stromberger 2005). Während der ökonomischen Krise in den 197oern zeigte das österreichische neo-korporatistische System eine überraschende politische und ökonomische Stabilität und rückte in den Fokus der nationalen und auch internationalen politikwissenschaftlichen Auseinandersetzung (Heinisch 2012, 2000; Katzenstein 1984, Kittel 2000, Luther/Müller 1992, Müller 2006, Sweeny/Weidenholzer 1988, Traxler 1993). Vor allem die Sozialpartnerschaft als Garant für die „ultrastability“ weckte das Interesse (Gerlich/Grande/Müller 1988). Aus diesem Grund ist die von Katzenstein im Jahr 1984 gestellte Frage noch immer aktuell: „How can this political management of the economy and the institutionalization of political conflict work so smoothly without sanctions?" (Katzenstein 1984: 70).

Speziell seit den 1990er Jahren hat eine Reihe von Änderungen der sozialen, politischen und ökonomischen Rahmenbedingungen die „Blütezeit“ der Sozialpartnerschaft beendet. Neben dem Abbau des machtpolitischen Duopols Sozialdemokratische Partei Österreichs (SPÖ) - Österreichische Volkspartei (ÖVP) sind sozioökonomische Gründe (Stichwort Globalisierung) genauso $\mathrm{zu}$ nennen wie Entscheidungsverlagerungen auf eine supranationale, europäische Ebene sowie ein Wandel der politischen Kultur in Österreich. Als Folgen dieser Entwicklungen wurde ein veritabler Verlust der politischen Einflussnahme der Sozialpartner bis hin zu einem Bedeutungsverlust von einstmals politisch zentralen und informellen Gremien festgestellt (Karlhofer/Tálos 2000, Tálos 2006, Tálos/Stromberger 2005, 2004; Traxler 1996) ${ }^{1}$. Angesichts dieser Entwicklungen und vor allem

1 Kittel und Tálos zeigen in ihrem 2001 veröffentlichten Buch „Gesetzgebung in Österreich“, dass es nie eine „Allmacht“ der Sozialpartner gab. Die größten Einflussmöglichkeiten waren immer nur in ihren Kernbereichen (Arbeit, Soziales, Arbeitsmarkt) vorhanden. Die Autoren zeigen an Beispielen auf, dass die Sozialpartner selbst in „ihren“ Themenfeldern nicht immer in den Gesetzgebungsprozess eingebunden wurden. nach der Bildung einer Mitte-rechts-Regierung von ÖVP und Freiheitlicher Partei Österreichs (FPÖ) im Jahr 2000 wurde ein weiterer Verlust von politischer Einflussnahme beziehungsweise ein Rückgang der Gestaltungspotenziale der Sozialpartnerschaft und des Austrokorporatismus von der österreichischen Politikwissenschaft konstatiert (Karlhofer 2005, Karlhofer/ Tálos 2006, 2000; Tálos 2006, 2015, Tálos/Stromberger 2004, 2005). Dies führte auch zu einer generellen Infragestellung der bis dahin als „common sense“ angesehenen Einordnung des österreichischen politischen Systems als korporatistisch geprägter Konkordanzdemokratie (Stromberger/Tálos 2005) beziehungsweise zur Feststellung eines Wandels von einem Nachfragezu einem Angebotskorporatismus (Krings 2013, Traxler 1993).

Gerade im zentralen arbeitspolitischen Einflussbereich der Sozialpartnerschaft (Tálos/Kittel 2001, Tálos/ Stromberger 2004) kam es jedoch bereits während der als Zäsur der Sozialpartnerschaft zu beschreibenden Zeit zwischen den Jahren 2000 und $2006 \mathrm{zu}$ einem Wiederaufschwung (etwa der Beschluss zur „Abfertigung Neu“), einer Re-Vitalisierung (wie die Verhandlungen und der Beschluss über die Novelle zum Arbeitszeitgesetz 2007) und zu einem Zugewinn der sozialpartnerschaftlichen Einfluss- und Machtpotenziale unmittelbar nach der ÖVP/FPÖ/BZÖKoalitionszeit (Besetzung der Schlüsselministerien durch Sozialpartner in der Wiederauflage der Großen Koalition sowie der Beschluss von Kurzarbeitsmaßnahmen während der Finanzkrise). Daran anknüpfend zeigte Österreich während der Banken- und Finanzkrise eine in Europa vergleichsweise gute ökonomische Performance (Eichhorst/Weishaupt 2013, Flecker/ Hermann 2015). Darüber hinaus verabschiedete die Regierung in Akkordierung mit den Sozialpartnern eine Reihe von Gesetzen, die in einem europäischen Kontext von Rezession, Austerität und Sozialabbau zu beurteilen sind, wie etwa die Einführung einer bundesweit geregelten „Bedarfsorientierten Mindestsicherung“ sowie die Verabschiedung des „Sozial- und Lohndumpinggesetzes“. Das spezifisch österreichische korporatistische Setting ist daher erneut in den Fokus der (internationalen) politikwissenschaftlichen Forschung gerückt (Afonso 2013, Afonso/March 2010, Afonso/Papadopoulus 2013, Leibrecht/Rocha-Akis 2014, Paster 2014, Pernicka/Hefler 2015, Torpe 2014, Wineroither 2013). In diesem Kontext zeigt sich, dass neokorporatistische Strukturen auch in einer international vergleichenden Perspektive stabil (Afonso/ 
Papadopoulus 2013) und für Regierungen interessant zu sein scheinen (Baccaro/Simoni 2008). Mit anderen Worten: Sie sind „back in vogue“ (Culpepper 2002: 774). Zusammengefasst ließen tiefgreifende Zäsuren des Austrokorporatismus und zunehmende Bedeutungsverluste der Sozialpartnerschaft den Schluss zu, dass die Regierungskonstellation von ÖVP und FPÖ nun $\mathrm{zu}$ einem Ende des bekannten Musters der korporatistischen Interessensakkordierung, politischen Mitgestaltungslogik und vor allem zu einem Ende der Einbindung in Gesetzwerdungsprozesse führen würde. Die Sozialpartnerschaft in Österreich erlebte jedoch ab dem Jahr 2006 auf „(...) verschiedenen Ebenen eine Wiederbelebung (...)“(Tálos 2008: 124).

So

„(...) haben die Beispiele Abfertigung (2001), die Neuregelung der Zumutbarkeitsbestimmungen in der Arbeitslosenversicherung (2004) und zuletzt die Einigung über das sogenannte Arbeitszeitpaket (2007) gezeigt, dass eine Akkordierung der Interessen mit anschließender Rezeption durch Regierung und Parlament auch weiterhin - wenn auch bei weitem nicht mehr in dem Ausmaß wie in vergangenen Jahrzehnten - zustande kommt. " Karlhofer 2007: 400)

Gerade in arbeitspolitischen Bereichen bestehen weiterhin beträchtliche Akkordierungs- und Einbindungsspielräume für die Sozialpartner (Karlhofer 2007, Tálos 2015, 2008) und die Wiederauflage der Großen Koalition wird als „sozialpartnerschaftliches Revival“ (Krings 2013) dargestellt. Ausgeblieben ist bisher, zu untersuchen, warum es in der sozialpartnerschaftlichen Zäsurzeit (Start der Kleinen Koalition 2000 und der Wiederauflage der Großen Koalition im Jahr 2007) nicht zu einem Ende, sondern sogar zu einer Revitalisierung sozialpartnerschaftlichen Einflusses gekommen ist. Um diese Lücke zu schließen, möchte das Papier die folgende Fragestellung beantworten: Was sind Erklärungen für die Kontinuität und Stabilität der Sozialpartnerschaft am Beispiel der Zäsurzeit zwischen 2000 und 2006?

Ausgehend von akteursbezogenen institutionellen Ansätzen werden die Präferenzen, Wahrnehmungen und Einstellungen der sozialpartnerschaftlichen Akteure in einer prekären politischen Periode zwischen den Jahren 2000 und 2006 untersucht. Dafür werden zwei Gesetzgebungsprozesse (verhandelt und beschlossen zwischen 2001 und 2007) im genuin sozialpartnerschaftlichen Feld der Arbeitspolitik analysiert. Dahinter steht das Ziel, Erklärungsansätze der Kontinuität und Stabilität der Sozialpartnerschaft außerhalb der politisch-rechtlichen Rahmenbedingungen zu erheben. Die Analyse dieser spezifischen Zeit der Zurückdrängung und des Wiederaufschwungs will nicht nur Erklärungen für die Stabilität der Sozialpartnerschaft aufzeigen. Sie soll auch einen Beitrag für die zukünftige Entwicklung der Sozialpartnerschaft angesichts der aktuellen und bevorstehenden innenpolitischen Lage liefern.

Nach der einleitenden Kontextualisierung des Themas sowie der Formulierung der Fragestellung wird in einem folgenden Schritt der theoretische Rahmen der Arbeit vorgestellt. Im nächsten Kapitel wird der methodische Zugang sowie die beiden für die Untersuchung relevanten Gesetzwerdungsprozesse beschrieben, um im darauf folgenden Abschnitt Erklärungsansätze für die Stabilität und Kontinuität sozialpartnerschaftlichen Einflusses am Beispiel der Jahre 2000-2006 abzuleiten. Das Fazit und ein Ausblick schließen die Arbeit ab.

\section{Theorie}

Ausgehend vom klassischen Institutionalismus hat sich der Neo-Institutionalismus als aussagekräftiger theoretischer Analyse- und Erklärungsrahmen wohlfahrts- und sozialstaatlicher Politik erwiesen, der den institutionell-politischen Ansätzen zuzurechnen ist. Gerade bei der Untersuchung korporatistischer politischer Arrangements genügt es weder, Entscheidungen und Handlungen ausschließlich auf institutionelle Pfadabhängigkeiten herunterzubrechen, noch einem rein auf rationales Handeln von Akteuren ausgerichteten Erklärungsmuster zu folgen: Die Interaktion von durch Institutionen bestimmtem Akteursverhalten und durch Akteursverhalten gebildeten Institutionen-Ausprägungen bildet die Basis des gewählten Forschungszugangs. Für den österreichischen Kontext haben Kittel und Tálos (2001) sowie Tálos und Stromberger (2004) mit Hilfe des akteurszentrierten Institutionalismus Analysen zur Einfluss- und Machtposition der Sozialpartnerschaft publiziert. Der Ansatz rückt das Akteursverhalten in den Vordergrund, doch im Gegensatz zu reinen „Rational-Choice“-Ansätzen wird davon ausgegangen, dass zwischen Akteur und Institutionen ein für die Analyse wohlfahrtsstaatlicher Kontexte zentraler Zusammenhang besteht: Akteursverhalten wird bestimmt von institutionenimmanenten Pfadabhängigkeiten. Umgekehrt bestimmen jedoch auch die Erwartungen, Präferenzen und Wahrnehmungen die Struktur und Ausrichtung der Institutionen. Es „(...) steht das Wahlhandeln der Akteure im Vordergrund, 
allerdings normativ geprägt und strategisch begrenzt durch institutionelle Regelsysteme“ (Czada 2004: 57).

Es geht demnach darum, Pfadabhängigkeiten sichtbar zu machen, die nicht nur für das Funktionieren, sondern auch für die Stabilität dieses neokorporatistischen Settings verantwortlich sind. Dafür stehen anlehnend an Tálos/Kittel (2001) verschiedene mögliche Akteurskonstellationen als Rahmen der Analyse zur Verfügung. Für den Austrokorporatismus sind vor allem (i) Konzertierung und Akkordierung (nur Dachverbände, bipartistisch), (ii) Konzertierung und Akkordierung (Regierung und Dachverbände, tripartistisch) sowie (iii) Akkordierung zwischen Regierung und einem Dachverband von Relevanz. Darauf aufbauend lassen sich die Einfluss- und Entscheidungsabläufe in Gesetzgebungsprozessen bestimmten Politiknetzwerken zuordnen, wobei die bedeutendsten corporatist concertation und sectoral corporatism sind: Zwischen dem Ende der 196oer und der 1990er Jahre können 19 von 22 für diesen Kontext relevante verabschiedete Gesetze dem Typus corporatist concertation und drei dem Typus sectoral corporatism zugeordnet werden (Tálos/Kittel 2001, Tálos/Stromberger 2004).

Um die Entscheidungsgründe der Akteure noch stärker in den Fokus zu rücken, lässt sich der akteurszentrierte Ansatz um entscheidungstheoretische Inputs erweiterten. Als Theorie-Add-ons fungieren die Theorie der Angemessenheit (logic of appropriateness) beziehungsweise die der Konsequenz (logic of consequence): Handeln orientiert sich an Regeln und institutionellen Rahmenbedingungen, die von Erwartungssicherheiten geprägt sind ${ }^{2}$, „(T)they guide behavior and stabilize expectations“ (March/Olsen 2009: 5) „[and prescribe] (...) more or less precisely, what is appropriate action" (March/Olsen 2009: 7). Dieser Logik folgend leiten Regeln das längerfristige Ziel von politischen Organisationen an, „(...) how to combine unity and diversity and craft a cooperative system out of a conflictual one" (March/Olsen 2009: 11). ${ }^{3}$ March und Olsen (2009, 1998) ziehen bei ihrer Gegenüberstellung der beiden theoretischen Ansätze zur Erklärung von politischem Handeln

2 In diesem Kontext weist Scharpf (2000) darauf hin, dass dieser Logik folgend in der Literatur bereits mehrmals festgestellt wurde, dass Geschichte nicht notwendigerweise effizient sei.

3 Hier lässt sich auch an Piersons "negativity bias“ anknüpfen. Er geht davon aus, dass für die Erhaltung des Status quo paradoxerweise größere Verluste hingenommen werden, als eine mögliche Veränderung des Ist-Zustand gebracht hätte (Pierson 1996). folgenden relevanten Schluss: Sind die Auswirkungen von (institutionellen) Identitäten und ihren impliziten Regeln klar, die Auswirkungen der Konsequenzen, Ressourcen und Einstellungen von zukünftigen Handlungen unsicher oder schwer abschätzbar, ist ein Handeln nach Überlegungen der Logik der Angemessenheit zu erwarten. Regeln - und damit lässt sich wieder gut an die akteurszentrierten Ansätze anknüpfen - beschreiben, was eine angemessene Aktion ist (March/Olsen 2009: 7), sie konstituieren Identitäten und „übersetzen“ institutionelle Settings in Orientierungen für das Handeln.

In tradierten, stabilen Settings von Interaktionsmustern, Regeln und Identitäten, wie es die österreichische Sozialpartnerschaft ist, scheint also eine rein rationale Handlungsgrundlage, die einer „Logik der Konsequenz" folgt, als unwahrscheinlich. Stattdessen macht es, March und Olsen (2009, 1998) folgend, in Hinblick auf die kollektive Entscheidungsfindung, den Stand der Ressourcen und den in der Sozialpartnerschaft zentralen Aspekt der Planbarkeit von politischen Entscheidungen mehr Sinn, das Handeln der in diesem Paper behandelten Akteure mit der „Logik der Angemessenheit“ zu analysieren. Hier spielen vor allem sogenannte „Erwartungssicherheiten“ eine zentrale Rolle, die sich aus den institutionellen Pfadabhängigkeiten ableiten, welche die Akteure anleiten und gleichzeitig auch von diesen gefördert werden.

\section{Methode und Fälle}

\subsection{Methode}

Die Sozialpartnerschaft beziehungsweise der Austrokorporatismus beschreibt ein spezifisches Muster der Interessenspolitik beziehungsweise -vermittlung, das durch Dachverbände der ArbeitnehmerInnen sowie der ArbeitgeberInnen und der Regierung (Tripartismus) getragen wird. Dieses System beruht auf der kooperativen und konzertierten Akkordierung von Interessen auf einer politischen Makroebene (Tálos 2006). Pernicka und Hefler (2015) definieren dahingehend vier institutionelle Felder der politischen Ökonomie, in denen traditioneller sozialpartnerschaftlicher Einfluss und Gestaltungsspielraum konstatierbar ist: Sozial- und Wirtschaftspolitik, kollektive Lohnverhandlungen, Arbeitsbeziehungen auf Betriebsebene sowie berufliche Aus- und Weiterbildung. Für diese Untersuchung wurde der Fokus auf arbeitspolitische Themenfelder bzw. arbeitspolitische Gesetzwerdungs- 
prozesse gelegt, die ein zentrales Feld der sozialpartnerschaftlichen Einflussnahme darstellen (vgl. etwa Tálos/ Kittel 2001). Für die Beantwortung der Fragestellung wurden zwei Gesetzgebungsprozesse zur Mitarbeitervorsorge („Abfertigung Neu“) (2002) ${ }^{4}$ und die Novelle zum Arbeitszeitgesetz $(2007)^{5}$ als Fallstudien analysiert. Beide Gesetzesmaterien wurden in der Übergangszeit zwischen dem in der Literatur konstatierten Bedeutungs- und Einflussverlust der Sozialpartnerschaft und einem erneuten Erstarken beziehungsweise Revival der Sozialpartnerschaft (Tálos 2008, 2015; Krings 2013, Hinterseer 2014) verhandelt und beschlossen.

Beim Gesetzwerdungsprozess „Mitarbeitervorsorge Neu“ stellte sich die sozialpartnerschaftliche Einflussnahme anders dar, als in einer genuin sozialpartnerschaftlichen Materie üblich. Die Verhandlungen $\mathrm{zu}$ diesem Gesetz fanden in einem völlig neuartigen politischen Setting statt, bei dem die SPÖ nicht in der Regierung vertreten war und sich die zwei Sozialpartner Arbeiterkammer (AK) und Gewerkschaftsbund (ÖGB) mit einer vollkommen neuen politischen Situation konfrontiert sahen.

Der Gesetzwerdungsprozess zur Novellierung des Arbeitszeitgesetzes startet in einer Zeit, wo die Sozialpartner nach einer ersten intensiven Zurückdrängung (Stichwort Pensionsreform 2000) wieder ihren Mitgestaltungsspielraum ausweiten konnten. Die Grundlagen zum Gesetz wurden schon während der Zäsur- und Oppositionszeit nach traditionellen Mustern der sozialpartnerschaftlichen Einbindung verhandelt. Beide Gesetzwerdungsprozesse repräsentieren also eine bedeutende Zeit für die Analyse der Stabilität der Sozialpartnerschaft und des neo-korporatistischen Arrangements in Österreich.

Hinzu kommt, dass in den für dieses Papier relevanten arbeitspolitischen Bereichen keine pluralistische Einflussnahme auf Gesetzwerdungsprozesse herrscht. Im stabilen korporatistischen Modell, vermittelt über die Sozialpartnerschaft, stellen Arbeiterkammer, Gewerkschaftsbund, Wirtschaftskammer sowie Landwirtschaftskammer die einzigen und relevanten Player dar. Dies ist nicht zuletzt dem institutionellen Set-up geschuldet, das von einer Quasi-Monopolstellung des Österreichischen Gewerkschaftsbundes über die Pflichtmitgliedschaft in den Kammern bis hin $\mathrm{zu}$

4 Betriebliches Mitarbeitervorsorgegesetz (Abfertigung neu)/BGBL 100/2002.

5 Novellierung des Arbeitszeitgesetzes/BGBL $61 / 2007$ privilegierten Begutachtungs- und StellungnahmeRechten dieser Gruppen reicht. So zeigt etwa Paster (2014) mit einer Untersuchung der Wirtschaftskammer in Österreich und ihrer Rolle während der ÖVP-FPÖRegierungszeit beim Abbau von sozial- und wohlfahrtsstaatlichen Maßnahmen, dass dieses „institutional setup“ ausschlaggebender für die Handlungserklärung ist als andere Erklärungsmuster. Durch ein process tracing (George/Bennett 2005) dieser Gesetzgebungsprozesse sollen daher Erklärungen für die Stabilität der Sozialpartnerschaft aufgezeigt werden. Dabei stellen die beiden ausgewählten Fälle keine vergleichende „small-n analysis“ im engeren Sinn dar. Vielmehr geht es um die tiefere Analyse zweier für den Untersuchungszeitraum relevanter Fälle, wobei Fall „(...) as an instance of a class of events“" (George/Bennett 2005, 17) definiert wird.

Die empirische Erhebung erfolgte durch eine Orientierung am qualitativen Forschungsparadigma. In der vorliegenden Forschungsarbeit wurden aufgrund der forschungsleitenden theoretischen Basis des akteurszentrierten Institutionalismus halb-strukturierte Interviews sowie forschungs- und interviewleitende Kategorien (siehe Anhang 1) konzipiert. Damit grenzt sich dieser qualitative Zugang von anderen, sehr offen gehaltenen Forschungszugängen innerhalb dieses Forschungsparadigmas ab (zum Beispiel "grounded theory“). Der Einsatz von qualitativen ExpertInnenInterviews wurde als brauchbare Methode angesehen, um die zentrale Analyse von Wahrnehmungen und Präferenzen zufriedenstellend vorzunehmen. Auf Basis dieser deduktiven Kategorienbildung wurde in jedem Interview ein Set gleicher Fragen gestellt. Damit konnte das nötige Maß an Vergleichbarkeit von Aussagen erreicht werden. Weiters waren die Interviews so offen konzipiert, dass genug Raum für alternative Gesprächsverläufe vorhanden war. Einschränkend bei diesem Zugang muss angemerkt werden, dass InterviewpartnerInnen mit einer großen Nähe zu politischen Parteien und Institutionen dazu neigen, Fragen in diesen wichtigen tiefergehenden Aspekten auszuweichen, um ein konsensuales und kooperatives Klima bei der Beantwortung von Fragen aufrecht zu erhalten.

Um eine tiefergehende Analyse der akteurszentrierten Stabilitätsfaktoren $\mathrm{zu}$ erhalten, wurde entschieden, vorrangig politische VertreterInnen der Sozialdemokratischen Partei, der Arbeiterkammer und des Gewerkschaftsbundes zu befragen. Daneben wurden aber auch Interviews mit VertreterInnen der Volkspartei, der Wirtschaftskammer und der Frei- 
heitlichen Partei abgehalten. Durch die politische Situation der ÖVP-FPÖ-Regierung und den damit verbundenen Schockzustand und die politische Neuorientierung der SPÖ, der AK und des ÖGB konnten in diesem Bereich neue und relevante Erkenntnisse erwartet werden. Die als semi-standardisierte Interviews konzipierten Befragungen bestanden aus zwei Abschnitten. Im ersten wurden Fragen zum Ablauf und zur Einschätzung der beiden Gesetzwerdungsprozesse gestellt. Die Fragen im zweiten Teil der Interviews zielten auf die Einschätzungen, Präferenzen und Wahrnehmungen der Akteure zur sozialpartnerschaftlichen Gestaltungs- und Einflussmöglichkeit auf politische Prozesse sowie der korporatistischen Zusammenarbeit beziehungsweise der Konzertierung und Akkordierung der einzelnen Verbände sowie der Regierung ab. Die Auswahl erfolgte vorwiegend durch ein „Schneeballsystem“, also durch Empfehlungen und vermittelte Termine der interviewten Personen. Dieses Sampling-Verfahren ist gerade bei Eliten- oder ExpertenInneninterviews im Rahmen von Fallstudien ein zielführendes Verfahren: So können Personen eruiert werden, welche sich durch die vorbereitenden Recherchen nicht erschlossen haben. Sie zeichnen sich durch relevantes Wissen aus, das sie durch eine spezifische Einbindung oder eine spezielle Rolle während des Gesetzwerdungsprozesses erlangt haben (Bernacki/Waldorf 1981). Das „bias“-Problem muss jedoch ständig reflektiert werden: Warum empfiehlt jemand genau diese Person und warum nicht jemand anderen? Wie wird ein Minimum an Repräsentativität durch Geschlecht, Alter, Position, Stellung etc. garantiert? Trotzdem bietet sich bei einer an Präferenzen und Wahrnehmungen innerhalb institutioneller Rahmenbedingungen orientierten Forschung eine empirische Erhebung mit qualitativen Interviews an (siehe etwa auch Tálos/Kittel 2001 und Tálos/Stromberger 2004). Unterstützt wurde die Rekonstruktion und Analyse der Gesetzwerdungsprozesse mit Hilfe einer Dokumentenanalyse von medialer Berichterstattung, Stellungnahmen der Sozialpartner und stenografischen Protokollen. Die Analysen der Gesetzwerdungsprozesse wurden in Anlehnung an das theoretische Fundament dieses Papiers in folgende Matrix überführt:

Tabelle 1: Analysematrix für die „Abfertigung Neu“ im Jahr 2002 und für die Novelle zum Arbeitszeitgesetz im Jahr 2007

\begin{tabular}{lll}
\hline & \multicolumn{1}{c}{ Abfertigung Neu 2002 } & \multicolumn{1}{c}{ Arbeitszeitgesetz 2007 } \\
\hline Anstoß/Anlass & Regierung, dann Sozialpartner & Regierung an Sozialpartner \\
Verteilung & distributiv & regulativ \\
Opposition & $\begin{array}{l}\text { keine; bzW. Versuch der Regierung, Sozialpartnerschaft } \\
\text { zu schwächen }\end{array}$ & keine \\
Einbindung der & ja; WKO, ÖGB, AK; aber nicht konstruktiv, sondern & ja; WKO, ÖGB, AK \\
Dachverbände & destruktiv orientierte Einbindung & \\
Akkordierung der & teilweise; Einfluss über Dachverbände gegeben & ja \\
Dachverbände & Verhandlung; hierarchische Abstimmung durch & Verhandlung; hierarchische Abstimmung durch \\
Handlungssituation & Abschluss des Verhandlungsprozesses durch die & Abschluss des Verhandlungsprozesses durch die \\
& Präsidenten & Präsidenten \\
Kkteurskonstellation & Konzertierung und Akkordierung (bipartistisch und & Konzertierung und Akkordierung (tripartistisch) \\
& tripartistisch) &
\end{tabular}

Anmerkungen: WKO: Wirtschaftskammer Österreich,ÖGB: Österreichischer Gewerkschaftsbund, AK: Kammer für Arbeiter und Angestellte Österreich 


\subsection{Fälle}

\subsubsection{Mitarbeitervorsorge (2002)}

Hier handelt es sich um ein Gesetz, bei dem trotz einer ÖVP-FPÖ-Koalition die Sozialpartner Arbeiterkammer und Gewerkschaftsbund eingebunden wurden, wenn auch, wie hier gezeigt wird, unter stark geänderten Vorzeichen. Vergleicht man diesen Gesetzwerdungsprozess etwa mit den Pensionsreformen der Jahre 2000 und 2003, so erscheint die Sozialpartnereinbindung verwunderlich: Die sozialpartnerschaftlichen Interessensvertretungen der ArbeitnehmerInnenseite wurden von den bisher in der 2. Republik üblichen Einflusskanälen abgeschnitten, was einen massiven Bruch der tradierten Abläufe in Gesetzwerdungsprozessen darstellte. Dies unterstreichen auch die für den österreichischen Kontext intensiven Streikmaßnahmen. Die Einbindung der Sozialpartner folgte also nicht der traditionellen oder klassischen Einbindungslogik. Vielmehr war es eine strategische Einbindung mit einem „Destruktionsziel“ (Interview 4).

„Wenn man das den Sozialpartnern übergibt, dieses

Paket, das führt dazu, dass deren Handlungsunfähigkeit

irgendwie demonstriert wird: Die werden sich in dieser

Frage nicht einigen. "(Interview 4$)^{6}$

Diese Vorgangsweise war ein absolutes Novum und stellte bis dahin bekannte Verhandlungs- und Koordinierungslogiken ins Abseits. Die Sozialpartner fanden auch zu keiner Einigung aufgrund von Differenzen bei den Finanzierungsdetails sowie den Ansprüchen (vgl. z.B. Nationalrat 2001) und die Verhandlungen zwischen Arbeiterkammer, Gewerkschaftsbund und Wirtschaftskammer scheiterten. Die Lösung brachte schlussendlich eine „Präsidentennacht“ zwischen den Präsidenten Fritz Verzetnitsch (ÖGB) und Christoph Leitl (WKÖ): Ohne direkte Einbindung der Parteien SPÖ und ÖVP wurde hier über das neue Abfertigungsmodell verhandelt und letztendlich auch ein Gesetzespaket beschlossen. Das institutionelle Setting der Sozialpartnerschaft, arbeitspolitisch relevante Materien $\mathrm{zu}$ koordinieren, konnte auch unter veränderten politischen Rahmenbedingungen erhalten bleiben -

6 Diese Einschätzungen teilt der interviewte FPÖPolitiker nicht und sieht die von den InterviewpartnerInnen angesprochene Annäherung als "glatte Lüge“. Die Bezeichnung der Arbeiterkammer als „zahnloser Idiotenverein“ spiegelt jedoch eine kategorische Ablehnung dieser Verbändestruktur wider (Interview 10). wenn auch auf einer hierarchischen Ebene und unter neuen Vorzeichen der Verhandlungssituation (sectoral corporatism). In dieser für die sozialpartnerschaftliche Zukunft sensiblen Phase spielten Erwartungssicherheiten, gerade bei einer Aussicht auf Einigung, eine zentrale Rolle. Dies hat sich vor allem bei den befragten VertreterInnen der Fraktion Sozialdemokratischer GewerkschafterInnen (FSG), welche für die arbeitspolitische Ausrichtung der SPÖ bedeutend sind, gezeigt: Sie waren über diese Einigung sehr überrascht und mit zentralen Details unzufrieden. Trotzdem verkauften sie „nach außen“ die offizielle Erfolgsdarstellung der Wirtschaftskammer und des Gewerkschaftsbundes (ÖGB 2002, WKÖ 2002; siehe auch Nationalrat 2002, DiePresse.com 2002a, 2002b). Die intern schlechte Stimmung der unzufriedenen Mitglieder konnte mit der Aussicht auf eine zukünftige politische Mitgestaltung kontrolliert werden. Der Inhalt dieser Gesetzesmaterie kann als distributiv bezeichnet werden ${ }^{7}$, wodurch sich auch die schwierige Positionsfindung erklären lässt. Bei diesem Abfertigungsmodell werden Beiträge der ArbeitgeberInnen an die ArbeitnehmerInnen (indirekt über die Vorsorgekassen) ausbezahlt. Einerseits bestand gerade durch die in Österreich traditionellen Debatten über die (zu) hohen Lohnnebenkosten hier ein großer Konfliktpunkt. Das alte Abfertigungssystem fungierte für langjährig Beschäftigte als eine Art Kündigungsschutz, was wiederum aus Sicht der Unternehmen aufgrund der zu bildenden Rücklagen mit hohen Kosten verbunden war.

Die Diskussion um ein neues Abfertigungsrecht wurde von den Regierungsparteien ÖVP und FPÖ angestoßen. Einerseits durch eine Nicht-Einigung innerhalb der Regierung und andererseits den Versuch, mit der Übertragung dieser Materie an die Sozialpartner diese zu schwächen, kam den Regierungsparteien eine eher passive Rolle zu. In Bezug auf das MatrixLabel "Handlungssituation“ (siehe Tabelle 1) wurde gerade in diesem Gesetz die letztgültige Entscheidung für die Konzeption des Gesetzesvorschlags von den Präsidenten der Wirtschaftskammer und des Gewerkschaftsbundes entschieden. Wie weiter oben bereits skizziert, war das Ergebnis aus Sicht der ArbeitnehmerInnenvertretung in Bezug auf die beschlossenen Berechnungsgrundlagen mehr als überraschend und vorwiegend negativ, als Gesamtpaket jedoch positiv bewertet. Man kann bei der Entstehung des Gesetzes in Anbetracht der bis dahin traditionellen Einflusslo-

$7 \quad$ Kittel (2001) anlehnend an Beyme (1997). 
gik nicht von einer tripartistischen Akkordierung und Konzertierung sprechen: Die Wirtschaftskammer war mit der Regierungspartei ÖVP bipartistisch akkordiert, sie bildeten eine starke Achse (sectoral corporatism). Die der SPÖ nahestehenden Sozialpartner suchten, wahrscheinlich in Anbetracht ihrer misslichen und politisch neuartigen Lage, die Nähe beziehungsweise eine Akkordierung mit der Wirtschaftskammer. Diese wiederum war nicht uninteressiert an einer Akkordierung mit dem sozialpartnerschaftlichen Gegenüber: Die Aussicht auf die Wahrung sozialpartnerschaftlicher Einflusslogik machte eine Einigung aus Sicht aller Sozialpartner politisch wünschenswert. Die durchwegs positiven offiziellen Stellungnahmen des ÖGB, welche die negativen Bewertungen der befragten Akteure in diesem Fall konterkarieren, lassen sich aus diesem Kontext erklären.

\subsubsection{Arbeitszeitgesetz (2007)}

Bei der Änderung des Arbeitszeitgesetzes 2007 und dem Arbeitskräfteüberlassungsgesetz wurde zum ersten Mal im Gesetzgebungsprozess der 2. Republik nicht nur ein gemeinsames Positionspapier, sondern auch eine gemeinsame Stellungnahme zum Gesetzesentwurf (ÖGB/LWK/AK/WKÖ 2009, Reiter 2009) verfasst. Im Gesetz zur Änderung des Arbeitszeitgesetzes lässt sich eine Re-Vitalisierung des sozialpartnerschaftlichen Einflusses konstatieren: Der Text des Regierungsentwurfs gleicht dem des Sozialpartnerentwurfs sowie dem des beschlossenen Gesetzes. Bei diesem Gesetzgebungs- und Verhandlungsprozess lassen sich die vorhin beschriebenen Grundpositionen der Sozialpartner gut wiedererkennen. Auf der einen Seite setzen sich die ArbeitgeberInnenverbände mit ihren Forderungen nach längeren Normalarbeitszeiten durch, während die Interessensvertretung der ArbeitnehmerInnen in Anlehnung an das Arbeitskräfteüberlassungsgesetz ein Ausschöpfen des Flexibilitätsspielraums an eine kollektivvertragliche Vereinbarung binden konnte (Nationalrat 2007a, 2007b).

Wie in der Matrix (Tabelle 1) dargestellt, haben die Sozialpartner den Verhandlungsanstoß für eine Arbeitszeitflexibilisierung gegeben. Dies spiegelt sich auch in der bereits thematisierten Tatsache wider, dass in dieser regulativen Materie die Sozialpartnerschaftsvereinbarung der Regierungsvorlage sowie dem beschlossenen Gesetz gleicht. Die Untersuchungen haben jedoch gezeigt, dass man bei dieser Materie den Begriff „Anstoß“ differenziert betrachten muss: Diskus- sionen und Auseinandersetzungen um Arbeitszeit und ihre Flexibilisierung zwischen der Wirtschaftskammer/ ÖVP und dem Gewerkschaftsbund/SPÖ sind ein Kontinuum der industriellen und sozialpartnerschaftlichen Beziehungen in Österreich. Den Beschlüssen gingen jahrelange, sehr intensiv geführte Auseinandersetzungen zwischen den beiden politischen Gruppen voraus. Vor allem die Industriellenvereinigung hat mit ihren Vorstößen zu mehr Arbeitszeitflexibilität die Diskussion immer wieder angeheizt und die Wirtschaftskammer unter Druck gesetzt. Die Regierungsparteien ÖVP/FPÖ haben die Konflikte auf die Sozialpartnerschaftsebene verlagert, wo trotz Oppositionszeit die SPÖ über die ihr nahestehenden Interessensgruppen eingebunden war. So ist parallel zur Mitte-rechtsRegierung und mit der Neuauflage der Großen Koalition im Jahr 2006 eine Arbeitszeitnovelle entstanden. Die Dachverbände waren also in einer arbeitspolitisch höchst relevanten Materie - ungeachtet neuer Regierungskonstellationen und Parlamentsmehrheiten - akkordiert und konzertiert. Passend zu den theoretischen Überlegungen zu dieser Arbeit zeigt sich eine corporatist concertation als Form des Politiknetzwerks. Der Gesetzwerdungsprozess kann im Gesamtkonzept als tripartistische Akkordierung und Konzertierung zwischen Dachverbänden und Regierung und über weite Strecken als bipartistisch zwischen den Dachverbänden beschrieben werden.

Bei beiden dargestellten Gesetzesbeschlüssen waren es demnach die Präsidenten, die mit raschen Beschlüssen Lösungen für teilweise langjährige Verhandlungsprozesse herbeiführten. Dabei scheint es aber nicht so gewesen zu sein, dass die Präsidenten mit einem von ihren ExpertInnen und VerhandlerInnen ausgestatteten Mandat in die Verhandlungen gingen. Sie haben Lösungen herbeigeführt, die in den Reihen der sozialdemokratischen Akteure für Überraschung und Erstaunen sorgten (vgl. Interview 5 und 7). Diese Entscheidungsebene kann als „hierarchische Entscheidung" gesehen werden, die sich von den anderen Verhaltensmustern kollektiver Entscheidungsfindungen doch stark unterscheidet (vgl. Mayntz/Scharpf 1995, Scharpf 2000). Diese Handlungssituation kann mit einer Beschleunigungstendenz von politischen Entscheidungen erklärt werden. Möglich ist auch, dass die Parteien mit den Präsidenten eine engere Verbindung haben, um ihre politischen Vorstellungen durchzusetzen, und die Präsidenten wiederum die nötige Macht besitzen, weitreichende Entscheidungen letztendlich im Alleingang zu beschließen. Dies findet in der um 
den Aspekt der Handlungssituationen erweiterten Matrix von Tálos und Kittel Eingang. Es zeigt sich, dass das Label „hierarchische Entscheidung“ einen wichtigen und nicht zu unterschätzenden Einflussfaktor bei der Beurteilung der Entscheidungsfindung in diesem beobachteten Zeitraum darstellt.

\section{Erklärungsansätze für die Stabilität und Kontinuität der Sozialpartnerschaft in Zeiten der Zäsur}

Konsens herrscht bei den Befragten darüber, dass die Regierungsbildung von ÖVP und FPÖ im Jahr 2000 die größte Zäsur in der Geschichte der österreichischen Sozialpartnerschaft darstellte, dies deckt sich mit den Befunden der politikwissenschaftlichen Literatur. Erstmals in der 2. Republik war eine Partei mit ausgewiesener „Anti-Sozialpartnerschafts“-Haltung in der Regierung vertreten. Diese Haltung, die zur politischen Ausrichtung der FPÖ in dieser Zeit als Anti-System und Anti-Establishment-Partei passte, paarte sich mit dem Selbstverständnis der ÖVP unter der Führung von Schüssel, einen neuen „Politikstil“ in Österreich einzuführen. Dies manifestierte sich in einer Reihe von "schnell“ verabschiedeten Gesetzen, die ohne Einbindung der Sozialpartner beschlossen wurden (Stichworte Pensionsreformen, Ambulanzgebühr). In diesem Zusammenhang zeigte sich auch der Einfluss der SPÖ-Opposition auf die Struktur und Logik des Einflusses der Sozialpartnerschaft:

„Dann kommt entweder aus der Arbeiterkammer ein Gesetzesentwurf oder aus dem Ministerium ein Gesetzesentwurf. (...) Das ist sehr unterschiedlich und darum ist es wichtig, dass man möglichst überall bei diesen Entwicklungsschritten und Möglichkeiten dabei ist, dann kann man es auch beeinflussen. Weil wenn man da nicht dabei ist, dann kann man es auch nicht beeinflussen. "(Interview 5)

Die Frage, die sich natürlich stellt, ist, warum es die Sozialpartnerschaft geschafft hat, diese „Eiszeit“ zu überdauern beziehungsweise in letzter Konsequenz sogar gestärkt aus dieser Phase herauszukommen. Die vorliegenden Analysen zu den arbeitspolitischen Gesetzwerdungsprozessen „Abfertigung Neu“ und zum Arbeitszeitgesetz 2007 zeigen, dass auch hier die Kontinuität von über Jahrzehnte entwickelten Einflusspotenzialen, Abhängigkeiten und Machtansprüchen krisenresistent zu sein scheint. Für die Klärung dieses Phänomens lassen sich aus der Forschung drei Aspekte, die im Folgenden themastiert werden, hervorheben: erstens die Rolle der Wirtschaftskammer, zweitens die Rolle der Freiheitlichen Partei als Beispiel für Player außerhalb der traditionellen sozialpartnerschaftlichen Settings und schließlich drittens der Strukturkonservatismus der kollektiven Akteure als Stabilisierungsfaktor. Daraus lassen sich zusammenfassend Fundamente der Stabilität ableiten.

\subsection{Die Rolle der Wirtschaftskammer}

Die Betrachtung des ersten Aspekts zeigt, dass die Wirtschaftskammer unter der politischen Leitung von Christoph Leitl die Linie der ÖVP-FPÖ-Regierung, Gesetze in arbeitspolitischen Fragen dauerhaft „am Sozialpartner vorbei“ $\mathrm{zu}$ beschließen, nicht mitgetragen hat. Der interviewte ehemalige FPÖ-Minister bezeichnete in diesem Zusammenhang die Wirtschaftskammer als „den ärgsten Feind der Regierung“ (Interview 10). Dies passierte, obwohl gerade zu Beginn der politischen Wende die Wirtschaftskammer mit der Industriellenvereinigung eine neue Achse gebildet hatte, welche die Arbeiterkammer und den Gewerkschaftsbund im Gesetzwerdungsprozess außen vor ließ. Für die Wirtschaftskammer eröffneten sich durch das nun mögliche Übergehen der Interessensvertretungen der ArbeitnehmerInnen und der ihr nahe stehenden Partei neue Möglichkeiten: Viele Wünsche und langjährige Forderungen der „Wirtschaft“, wie etwa Senkung der Lohnnebenkosten, Abschaffung und Senkung von Steuern, weitreichende Änderungen im Arbeits- und Sozialgesetz (Stichworte Flexibilisierung, Arbeitszeiten etc.) hätten nun ohne Akkordierung mit dem politischen Gegner umgesetzt werden können. Die Frage ist nun: Warum hat die Wirtschaftskammer dieses neue Macht- und Gestaltungspotenzial, in Anbetracht des informellen Charakters des sozialpartnerschaftlichen Arrangements, nicht genutzt?

Laut der für dieses Papier verwendeten theoretischen Basis des akteurszentrierten Institutionalismus handeln Akteure mit einem bestimmten (normativen) Ziel innerhalb eines Gemeinwohl-orientierten institutionellen Settings. Diese institutionellen Rahmenbedingungen leiten das Handeln an - Handlungsmotive und Handlungsentscheidungen sind also nicht nur durch rationale beziehungsweise spieltheoretische Motive erklärbar. Der institutionelle Überbau hat einen zentralen Einfluss auf die Handlungen: Institutionen - wie hier die Sozialpartnerschaft - bestimmen die Präferenzen der Akteure. Mayntz und Scharpf (1995) weisen darauf hin, dass Akteure und Player, die in 
ein institutionelles Setting eingebunden sind, niemals ganz autonom handeln können - die Identitäten und Regeln schränken „Alleingänge“ massiv ein. Außerdem stützen institutionelle Strukturen bestimmte Interaktionsorientierungen und Akteure können in solchen stabilen Gefügen, wie es die Sozialpartnerschaft ist, Interaktionsorientierungen von anderen Akteuren erwarten. Die Konsequenzen und Auswirkungen eines Übergehens des sozialpartnerschaftlichen Gegenübers wären also nur kurzfristig abschätzbar gewesen. Die Regeln dieser institutionellen Rahmenbedingungen garantieren demnach einen chaosfreien Raum für die Verwirklichung der Ziele und ermöglichen wechselseitige Erwartungssicherheiten. ${ }^{8}$

Die ersten Auflösungserscheinungen zeichneten sich nach dem Spaltungsprozess in Knittelfeld innerhalb der Freiheitlichen Partei ab, und wie aus den Interviews hervorgeht, war die dünne Personaldecke beziehungsweise die Unerfahrenheit und Inkompetenz der FPÖ (Fallend/Heinisch 2015) offensichtlich. Daher waren die weiteren Konsequenzen in Hinblick auf die konsensorientierten Arbeitsbeziehungen in Österreich, die in der 2. Republik von der WKÖ als Interessensvertretung der „Wirtschaft“ stets getragen wurden, nicht klar definierbar. Dies betraf nicht nur den hier untersuchten Politikbereich, sondern den gesamten Modus der Sozialpartnerschaft. Die Orientierung an bekannten Mustern von Regeln, Rollen und Identitäten in einer mittelfristigen Perspektive schien hier als Argument für die ArbeitgeberInnenseite stärker zu wiegen, als mit kurzfristigen Erfolgen möglichweise mittel- und längerfristig Schaden anzurichten. Anlehnend an die von Mayntz und Scharpf (1995) offen gelassene Frage, ob Institutionen als abhängige oder unabhängige Variablen fungieren, kann in diesem Kontext Folgendes argumentiert werden: Das institutionelle Setting (structure) bedingt eine Reproduktion bekannter Muster der Akteure (agents) - selbst bei einem großen politischen „window of opportunity“, wie es die Wirtschaftskammer am Beginn der ÖVP-FPÖ-Koalition vorfand. So beschreibt Interviewpartner 5 seine Einschätzung dieser Phase der Sozialpartnerschaft und die Rolle der Wirtschaftskammer wie folgt:

„Man muss schon den historischen Kontext auch sehen, auch wenn die schwarzen Sozialpartner quasi mit der ÖVP im schwarz-blauen Boot indirekt mit drinnen waren, die Leute in der Wirtschaftskammer waren nicht

8 Siehe auch: March/Olsen (2009). glücklich mit Schwarz-Blau und innerhalb der ÖVP hat es natürlich Riesenprobleme gegeben, weil der Schüssel mag die Sozialpartner nicht." (Interview 5)

Das tradierte sozialpartnerschaftliche Muster ist stabil, das heißt mit anderen Worten: Das Ausscheren einzelner kooperativer Akteure ist möglich, jedoch unwahrscheinlich. Auch eine Anlehnung an eine Logik der Angemessenheit liegt nahe: Handeln orientiert sich an bekannten Regeln und institutionellen Rahmenbedingungen, die von Erwartungssicherheiten geprägt sind. Anlehnend an Mayntz und Scharpf (1995) und Scharpf (2000) kann gefolgert werden, dass die Leitlinie für Handlungsentscheidungen innerhalb eines institutionellen und kooperativen Settings stärker durch die Angst vor Verlusten gesteuert ist als durch die Aussicht auf mögliche (politische) Gewinne. Diese Angst vor dem längerfristigen Verlust von Einfluss und Macht in einer für die Interessensvertretung der Wirtschaftstreibenden wichtigen Institution (vgl. Interview 3, 4, 6) ist demnach ein bedeutender Erklärungsansatz für die Handlungsentscheidung. So hält Interviewpartner 3 von der Arbeiterkammer fest, dass „(...) die Wirtschaftskammer punktuell schon darauf geachtet hat, die Sozialpartnerschaft nicht einfach komplett zu beschädigen“. Es sind, wie die theoretischen Auslegungen akteurszentrierter Ansätze nahelegen, nicht nur die institutionellen Pfadabhängigkeiten und identitätsstiftenden Momente für die Erklärung von Handlungen heranzuziehen: Die persönliche Ablehnung Christoph Leitls gegenüber der FPÖ und dem Bündnis Zukunft Österreich (BZÖ) (Interview 1) stellte sozusagen eine wichtige lebenserhaltende Maßnahme der Sozialpartnerschaft dar. Dies unterstreicht auch die folgende Aussage:

„Die Sozialpartnerschaft lebt davon, dass die Partner einander respektieren, weil sie wissen, dass sie gemeinsam mehr erreichen können als gegeneinander. Daher kann es nicht das Bestreben von Sozialpartnern sein, entsprechende ,windows of opportunity' unmittelbar zu nutzen." (Interview 11)

Der ÖVP-Minister (Interview 12) hält in diesem Zusammenhang den wichtigen Aspekt fest, dass die Zurückdrängung der Sozialpartner deren Revival begünstigte:

„Die Versuche, einzelne Sozialpartner auszugrenzen, etwa neben $A K$ und ÖGB die Landwirtschaftskammern und die Raiffeisenorganisation zu schwächen, führte zu einer Solidarisierung und zu einem vertieften Bewusstwerden der wechselseitigen Abhängigkeiten." (Interview 12) 


\subsection{Die Rolle der FPÖ}

Diese durch institutionelle Pfadabhängigkeiten geprägten Präferenzen der sozialpartnerschaftlichen Akteure helfen auch die Rolle der FPÖ zu verstehen. Die anfänglichen Versuche der Regierung, mit dem Credo „speed kills“9 eine neue, sozialpartnerschaftsfreie Gesetzgebungskultur in Österreich zu implementieren, wurden schließlich von der FPÖ eingebremst. Die Freiheitlichen schienen vor allem nach der Einführung der ersten Pensionsreform Bedenken zu haben, ihre gerade gewonnene Wählerschaft zu verlieren. FPÖPolitikerInnen holten sich nach diesen Erfahrungen mit einem wichtigen, den Großteil der Bevölkerung betreffenden Gesetz in der Arbeiterkammer Wien Expertise und Rat für weitere geplante arbeitspolitische und arbeitsrechtliche Reformprozesse (Interview 3 und 4). In weiterer Folge suchten freiheitliche MandatarInnen Nähe zu den sozialdemokratischen VertreterInnen im Sozialausschuss des Parlaments, um mit ihnen vor den Ausschusssitzungen die von der ÖVP vorgelegten Gesetzesentwürfe durchzusprechen (Interview 6). Damit bekamen die zurückgedrängten ArbeitnehmerInnen-Interessensgruppen wieder Einfluss und gewannen an Bedeutung. Dies waren angesichts der ausgeprägten Anti-Establishment- und vor allem AntiSozialpartnerschaftshaltung der Freiheitlichen Partei doch überraschende Entwicklungen.

Afonso (2013) geht davon aus, dass es vor allem dann zu Konzertierungsbestrebungen der Regierung kommt, wenn (i) die Unterstützung in der Bevölkerung schwach ist beziehungsweise wenn sie (ii) „(...) react to politicisation processes by trade unions when issues become salient for voters“. Diese Feststellung hilft, die Annäherungen, nicht nur der FPÖ, sondern auch der ÖVP beziehungsweise der Regierung, an die Sozialpartnerschaft zu erklären. Es zeigt sich, dass gerade bei VertreterInnen von Interessensgruppen diese Erfahrungen der Zäsurzeit zu einem neuen Selbstverständnis und Selbstvertrauen in Bezug auf die aktuelle Situation sowie auf die mittelfristige Zukunft der Sozialpartnerschaft geführt haben. Es wird jedoch klar, dass die Entzauberung einer „Allmacht“ des Sozialpartnereinflusses von Tálos und Kittel im Jahr 2001 auch heute, selbst in Anbetracht eines gestärkten Auftretens der Sozialpartner, relevant ist. Diesen Aspekt

9 Ein vom ÖVP-Politiker Andreas Kohl geprägter Begriff zum neuen angestrebten Politikstil der ÖVP-FPÖRegierung Anfang der 20ooer Jahre. unterstreicht auch Interviewpartnerin 6, die in dieser Zeit eine wichtige politische Funktion im parlamentarischen Ausschuss für Arbeit und Soziales innehatte (Interview 6). Der Wirtschaftskammer-Repräsentant (Interview 11) untermauert diese Bestrebungen: „Tatsächlich war immer der Wunsch sichtbar, sich sozial zu präsentieren, auch um Angriffe auf die Regierung zu entkräften.“ Diese beiden Beispiele machen deutlich, dass nach der Zäsur im sozialpartnerschaftlichen System die längerfristige Kontinuität der Sozialpartnerschaft nicht untergraben wurde.

\subsection{Strukturkonservatismus}

Die Streiks im Jahr 2003 kennzeichnen ein Ausscheren vom sozialpartnerschaftlichen Muster der Arbeitsbeziehungen: der Pfad konsensualen Aushandelns und von Akkordierung politischer Entscheidungen wurde verlassen. Theoretisch ist politisches Handeln in arbeitspolitischen Fragen also ohne Akkordierung der Sozialpartner möglich, oder anders formuliert, Handeln ist auch außerhalb der bekannten institutionellen Einflusslogiken denkbar. Die Analyse der Befragung zeigt, dass diese Schock-Erfahrung aus Sicht der vormals eingebundenen Akteure weit über das hier untersuchte arbeitspolitische Feld hinausging: Das gesamte bisher bekannte System der Interessensakkordierung und vor allem der politischen Einflussnahme stand zur Disposition. Für die Legitimität und für die Akzeptanz in der Bevölkerung war dieser „Alleingang“ jedoch nicht nur aus Sicht der SPÖ, des ÖGB und der AK problematisch, sondern auch für die Freiheitliche Partei. Bezugnehmend auf die bereits aufgestellten Schlussfolgerungen schien auch für die Wirtschaftskammer ein Alleingang nicht die adäquate Lösung zu sein (vgl. auch Interview 11 und 13):

„Weil logischerweise auch der Sozialpartner auf Wirtschaftsseite ein Interesse haben muss, dass der Sozialpartner auf ArbeitnehmerInnenseite einen Einfluss hat, weil sonst würde er ja selber einflusslos. Also das ist irgendwie schon eine Überlebensfrage sozusagen auch für die andere Seite." (Interview 6)

Die angesprochene Phase der Zurückdrängung hat dazu geführt, dass das Aufrechterhalten des konsensualen Klimas als am passendsten für die Erreichung der mittel- und langfristigen Ziele eingeschätzt wurde. Die zentrale Bedeutung eines sozialpartnerschaftlichen Klimas unterstreichen vor allem die befragten Minister (Interviewpartner 8 und 12). Ein wichtiger Faktor beziehungsweise Ausgangspunkt für den Aufschwung 
der Sozialpartner sind demnach die Erfahrungen aus der ersten Pensionsreform, gerade wie schon thematisiert vonseiten der Freiheitlichen Partei:

$$
\text { „(...) meine Wahrnehmung war (...) dieser erste }
$$

Elan, die Sozialpartner da jetzt außen vor zu lassen, war verschwunden und eigentlich Politik as usual irgendwie in gewisser Hinsicht wieder angesagt, ist mir vorgekommen. 2005, 2006 und so (...): das große Ziel (...) Gesetzesvorbereitung zu verlagern, der war also aus meiner Sicht kaum mehr wirklich erkennbar." (Interview 4)

In diesem Zusammenhang muss auch die Rolle des Gewerkschaftsbundes als Veto-Spieler gegen eine Anti-Sozialpartnerschaftspolitik als sehr bedeutend für die weitere Entwicklung des Austrokorporatismus und des sozialpartnerschaftlichen Selbstverständnisses angesehen werden. In der Wahrnehmung der Interessensvertretungen und der SPÖ funktioniert die Sozialpartnerschaft - trotz der einschneidenden Erfahrungen zu Beginn des Millenniums und trotz des wichtigen Zusammenhangs von Regierungskonstellation und Einflussstärke der Sozialpartner - „(...) auch unabhängig von Regierungen (...)“ (Interview 2). So war etwa die Periode der Alleinregierung der Sozialdemokratischen Partei in den 1970ern und Anfang der 1980er Jahre auch die mächtigste und einflussreichste der Sozialpartnerschaft (vgl. Tálos 2008). Dies zeigt sich am steigenden Einfluss des Sozialpartnersystems schon während der Regierungszeit von ÖVP/FPÖ/ BZÖ. Auch das bis zum Jahr 2000 eher untypische, aber ab den 2oooer Jahren stark an Bedeutung gewinnende Verhandlungsmuster sectoral corporatism unter bipartistischer Einbindung (Tálos/Stromberger 2004) konnte daran schlussendlich nichts ändern. Vielmehr wurde selbst bei diesem Gesetzwerdungsprozess zur „Abfertigung Neu“ rasch an einer Orientierung an der bekannten Einfluss- und Einbindungslogik gearbeitet. Um diese Konstanz und auch den dahinter stehenden Strukturkonservatismus herauszuarbeiten, wurde bei den Interviews mit Akteuren von SPÖ, Gewerkschaftsbund und Arbeiterkammer immer nach der Einschätzung des sozialpartnerschaftlichen Klimas und Arbeitens im hypothetischen Fall einer SPÖGrüne-Regierung gefragt. ${ }^{10}$ Der institutionellen Logik folgend zeigt sich dies auch bei der Einschätzung der ArbeitnehmerInnen-Akteure, die im Falle einer SPÖGrüne-Koalition kein Ausbremsen oder Übergehen

10 Eine Regierung aus Sozialdemokratischer Partei und Freiheitlicher Partei als einzige alternative Reformpartnerschaft wurde nur im Interview 10 erwähnt. der ArbeitgeberInnen-Vertretung innerhalb der Sozialpartnerschaft andenken. Viel größer ist die Sorge oder das Bestreben, die Grüne Partei von dieser Kooperation und Akkordierung trotz koalitionärer Mehrheit zu überzeugen. Aus der Sicht der Sozialdemokratie, als auch bei ÖGB und AK, wird diese hypothetische Zusammenarbeit vor allem in Bezug auf programmatische Übereinstimmungen im Sozialbereich positiv gesehen. Es herrscht aber keine Euphorie im Sinne eines großen Aufbruchs:

„Also zu meinen, dass man da ein linkes Programm zustande bekommt, würde ich groß bezweifeln. Und was dann wäre, also eine rot-grüne Regierung hätte jedenfalls auch einen starken Gegenwind eben von ÖVP und Wirtschaftsseite." (Interview 3)

Aufgrund der oben dargestellten Erfahrungen einer Nicht-Äquivalenz zwischen Sozialpartnerschaft und Regierungskonstellation werden doch einige Einschränkungen beziehungsweise Bedenken geäußert. Ein „Vorbei-Regieren“ an der Wirtschaft scheint nicht zweckmäßig, im Gegenteil wird dies eher als unproduktiv angesehen. „Jedenfalls real politisch betrachtet ist ein Vorbeiregieren an der Wirtschaft wahrscheinlich unrealistisch“ (Interview 4). Interviewpartner 7 geht einen Schritt weiter: Um die Wirtschaftskammer nicht als Fundamentalopposition zu haben, müsse die SPÖ viel größere Zugeständnisse machen, als wenn sie ihr politisches Programm in einer Großen Koalition durchsetzen wollte. Diese Einschätzung macht sowohl die tief verwurzelte Kontinuität sozialpartnerschaftlicher Einflusslogik als auch die Machtposition der einzelnen Sozialpartner sichtbar. Der Logik der Angemessenheit folgernd zeigt sich, dass bekannte Identitäten und Erwartungssicherheiten möglichen neuen politischen Gestaltungspotenzialen eher vorgezogen werden. Es herrscht demnach keine Stimmung irgendeiner Form von „Rache“ oder „Heimzahlen“ an ÖVP oder WKÖ. Dies liegt vor allem an den oben dargestellten Erfahrungen aus der Oppositionszeit, die eben mit der weiter oben skizzierten Logik der Angemessenheit der sozialpartnerschaftlichen Handlungslogiken interpretiert wird (siehe folgendes Zitat):

„(...) Sozialpartnerschaft wird gelebt, es ist nicht etwas, was von oben diktiert wird, das wird in Österreich tatsächlich gelebt und das ist gut so und das ist auch ohne Parteien gut so, und deswegen glaube ich auch, dass bei Rot-Grün es nicht so wäre, dass die Wirtschaftskammer völlig abgemeldet wäre, weil im Hintergrund die Sozialpartnerschaft gut funktioniert und weil auch jede Regierung daran Interesse haben muss, sich auch mit dem 
Wirtschaftspartner gut $z u$ verstehen. Ich meine, eine Regierung kann zwar Rahmenbedingungen schaffen, aber Arbeitsplätze kann sie keine schaffen. Das schafft die Wirtschaft und das ist das Entscheidende." (Interview 2)

Die befragten VertreterInnen der Sozialpartner AK und ÖGB machen unisono deutlich, dass sie selbst mit einer SPÖ-Grünen-Regierungskonstellation weiterhin arbeits- und sozialpolitische Gesetze mit der „Wirtschaft“ koordinieren würden - wobei hier sicher stark zwischen ÖVP und WKÖ unterschieden (werden) wird. Diese Orientierung einer auf Regeln und Interaktionserwartungen basierenden Logik der Angemessenheit von Akteuren innerhalb eines institutionalisierten Settings liefert eine Erklärung für die bereits besprochene Entscheidungslogik der Wirtschaftskammer unter Präsident Leitl, für die Beibehaltung der Erwartungssicherheiten eines konsensualen Klimas der Arbeitsbeziehungen innerhalb des institutionellen Rahmens der Sozialpartnerschaft.

Interviewpartner 9, ein Sektionschef im BMASK, unterstreicht durch seine Sicht aus der Ministeriumsperspektive den angesprochenen Strukturkonservatismus. Auch er würde im hypothetischen Fall einer Rot-Grün-Koalitionsregierung eine Zusammenarbeit in arbeitsmarkt- und arbeitspolitischen Fragen schwieriger einschätzen. Diesen von Erwartungssicherheiten geprägten institutionellen Konservatismus der sozialpartnerschaftlichen Beziehungen bringt der Gewerkschafter und Nationalrat (Interview 7) treffend in Bezug auf die Erfahrungen und Auswirkungen der ÖVP-FPÖ/BZÖ-Regierungsperiode auf den Punkt:

„Da war die Wirtschaft nicht glücklich und die ArbeitnehmerInnen nicht glücklich. Vor allem hast $d u$ da ein Eskalationspotenzial drinnen, das glaub ich nicht, dass das einer abschätzen kann, wenn er nicht wirklich weiß, wie es rennt. Das heißt, du bist gut beraten, wenn du einen vernünftigen Dialog auch mit aller Härte führst. Und weitestgehend Übereinstimmung findest, weil das einfach wirklich ein Garant für den sozialen Frieden bei der Republik ist." (Interview 7)

\subsection{Fundamente der Stabilität und Kontinuität}

Die Analyse der Gesetzwerdungsprozesse mit Hilfe der Dokumentanalyse und vor allem der qualitativen Interviews zur „Abfertigung Neu“ und der Novelle des Arbeitszeitgesetzes 2007 demonstrieren eine große Kontinuität und vor allem Stabilität der Sozialpartnerschaft. Dafür sind vor allem institutionelle Pfadabhängigkeiten verantwortlich, die einen erheblichen
Einfluss auf das Verhalten der sozialpartnerschaftlichen Akteure haben. Dies wurde im vorliegenden Papier an den „Erwartungssicherheiten“ festgemacht. Mit diesem Zugang lassen sich Erklärungen dafür geben, warum die Sozialpartnerschaft die für diese Untersuchung wichtige Periode der politischen Zäsur zwischen den Jahren 2000 und 2006 nicht nur „überlebte“, sondern daraus sogar gestärkt hervorging. Die von ÖVP und FPÖ gebildete Regierung lagerte im Jahr 2001 das Gesetz „Abfertigung Neu“ (Mitarbeitervorsorge) an die Sozialpartner aus, mit dem Ziel, die Sozialpartnerschaft weiter zu schwächen. Danach zeigten sich jedoch eine Wiederbelebung sowie eine erneute Einflussmacht auf Gesetzgebungsprozesse und politische Entscheidungen durch das sozialpartnerschaftliche Setting. Das „window of opportunity“, die Sozialpartnerschaft nachhaltig zu schwächen, konnte von den Regierungsparteien wider Erwarten nicht genutzt werden.

Tálos und Stromberger (2004) zeigten, dass in den ersten Jahren der neuen politischen Konstellation der bis dahin typische Netzwerktypus corporatist concertation zunehmend vom sectoral corporatism abgelöst wurde. Darin wurde die deutliche Zäsur und Umbruchsphase des korporatistischen Gestaltungsund Einflusspotenzials im hier untersuchten Zeitraum deutlich. Der analysierte Gesetzgebungsprozess zur „Abfertigung Neu“ kam, wie in dieser Phase üblich, durch die Initiative der Regierung und startete genau in einem Netzwerk nach dem Muster der sektoralen Einbindung. Doch durch die in diesem Papier analysierten institutionellen und akteursbezogenen Strukturen und Einflussgrößen konnte gezeigt werden, dass es schon während der Verhandlungsphase zu einem Wechsel zu einer (tripartistischen) und „klassischen“ corporatist concertation-Einbindungslogik kam. Dies markierte einen Wendepunkt zwischen Zurückdrängung und Wiederbelebung der Sozialpartnerschaft in arbeitspolitischen Fragestellungen. Dafür waren eben tradierte, durch sozialpartnerschaftliche Identitäten geprägte Erwartungssicherheiten verantwortlich, die zu der gemeinsamen Ausarbeitung des Arbeitszeitgesetzes 2007 während der Zäsurzeit führten und in einer demonstrativ gemeinsamen Stellungnahme aller Sozialpartner zu eben diesem Gesetz gipfelten. Die hier beschriebenen handlungsleitenden Motive der Akteure scheinen auch in Zeiten der politischen Zäsur und potenzieller politischer Entmachtung ein starker Garant für den Weiterbestand dieses Systems und somit auch für die „ultrastability“ der Sozialpartnerschaft im Rahmen der Einflussnahme auf arbeitspolitische 
Gesetzwerdungsprozesse zu sein. Dies manifestiert sich, wie in diesem Papier gezeigt werden konnte, an der Rolle der Freiheitlichen Partei, dem Handeln des Wirtschaftskammer-Präsidenten Leitl und an einer starken strukturkonservativen Haltung aller im Rahmen der sozialpartnerschaftlichen Akkordierung involvierten Akteure.

In Bezug auf die besprochene einschlägige politikwissenschaftliche Literatur wird deutlich, dass, entgegen den Prognosen Anfang der 2oooer Jahre, die Sozialpartnerschaft noch immer als einflussreiches und gestalterisches Tool der industriellen Beziehungen sowie der Gestaltung von Arbeitspolitik fungiert: Die Phase der Zäsur hat trotz starker Einschnitte in die traditionellen Muster neo-korporatistischer Politikgestaltung zu Beginn der 2oooer Jahre nicht zu einer nachhaltigen Neu-Orientierung und Neu-Positionierung der Sozialpartnerschaft geführt. (Dagegen hatte etwa die Kammerkrise in den 1990er Jahren zu einem tiefer greifenden Wandel beim Sozialpartner Arbeiterkammer geführt.) Vielmehr wurde, gestärkt durch die Suche nach Stabilität und Erwartungssicherheiten, der „alte“ und bekannte Weg sozialpartnerschaftlicher Abstimmung in arbeitspolitischen Materien gewählt. Der Wiederaufschwung wurde durch die Besetzung von „Schlüsselministerien“ (aus Sicht der Sozialpartnerschaft) mit zwei sozialpartnerschaftlich geprägten Akteuren untermauert.

Diese - und das zeigen ausnahmslos alle Befragungen - pflegen einen traditionellen konsensualen Stil, der von wechselseitiger Akkordierung geprägt ist. Dieser „neue“ Stil der zwei stark sozialpartnerschaftlich geprägten Minister ist streng genommen nicht neu, sondern das Rückbesinnen auf bekannte Identitäten, Handlungsmuster sowie Netzwerke, die eben - und das unterstreichen die Interviews auf Ministerebene deutlich (Interview 8 und 13) - erwartungssichere Räume politischen Handelns schaffen. Bekannte Strukturen leiten das Handeln an und machen auch für Außenstehende ein klares Muster sichtbar. Diese Revitalisierung tradierter, bekannter und vor allem in der Wahrnehmung bewährter Strukturen muss als ein wichtiger Grund für die Renaissance der sozialpartnerschaftlichen Einflussnahmen in arbeitspolitisch relevanten Gesetzwerdungsprozessen gesehen werden. So weist Interviewpartner 13 im Rückblick auf die „Zäsurjahre“ darauf hin, dass aus seiner Sicht die Sozialpartnerschaft nie ernsthaft in Gefahr gewesen sei: Selbst dem Wortlaut des Regierungsübereinkommens von ÖVP und FPÖ aus dem Jahr 2000 habe „ein Papier“ zugrundegelegen, an dem die Spitzen der Sozialpartner mitgearbeitet hätten. Diese Einschätzung zeugt von einer Hartnäckigkeit der sozialpartnerschaftlichen Pfadabhängigkeiten im politischen System. Durch die Besetzung der für diesen Bereich relevanten Ministerien mit Sozialpartnern (Hundstorfer/Mitterlehner) wurden nicht nur alte Pfade wieder betreten, sondern sogar gepflegt und ausgebaut (Hinterseer 2014).

Wie also in der neo-institutionellen und akteursbezogen orientierten Erklärung von Politik festgehalten, sind kollektive Handlungen und Entscheidungsfindungsprozesse auch bei divergierenden und selbst bei widersprüchlichen Präferenzen der einzelnen Akteure möglich (Jahnsen 2000). Dies betrifft nicht nur die Akkordierungs- und Konzertierungsspielräume für die eigentlich diametral auseinander liegenden Interessensgruppen von Kapital und Arbeit. Dass das institutionelle Setting eine Klammerfunktion für diese unterschiedlichen Präferenzen und Wahrnehmungen politischer Konflikte besitzt, kann nicht hoch genug geschätzt werden. Gerade in der Logik kollektiver, konsensorientierter Entscheidungsfindung zwischen zwei „Gegnern“ ist die Einigkeit in der eigenen politischen Gruppe zentral. Daraus lassen sich in weiterer Folge auch die noch immer beträchtlichen personellen Verflechtungen von Sozialpartnern, NationalrätInnen und MinisterInnen erklären (siehe etwa Ennser-Jedenastik). In tradierten, stabilen Settings von Interaktionsmustern, Regeln und Identitäten, wie es die österreichische Sozialpartnerschaft ist, folgen die Handlungslogiken der Akteure der Logik der Angemessenheit. Die daraus resultierende (wiedererlangte) Stabilität und Kontinuität unter neuen Vorzeichen (Tálos 2015) der politischen Einflussnahme sind Garant für die auch heute noch außergewöhnliche Stellung der Sozialpartnerschaft im Rahmen der industriellen Beziehungen.

"In general, since the formation of the new 'grand coalition' government replacing the conservative-populist predecessor government, the role of the social partners has significantly changed. They are now again strongly involved in all socio-economic matters of public interest, and in particular organised labour has given up its fundamental opposition towards the government by shifting into a policy line of smooth cooperation. " (Adam 2008)

\section{Fazit und Ausblick}

Gerade ab den 1980er Jahren kam es durch verschiedenste politisch-ökonomische Entwicklungen zu nachhaltigen Veränderungen des politischen Systems 
Österreich: Zu nennen sind vor allem das Aufbrechen des Duopols der Großen Koalition, das Aufkommen von neuen BürgerInnen-Bewegungen, das Erstarken der FPÖ, eine diversifiziertere Parteienlandschaft im Parlament, der EU-Beitritt Österreichs, eine zunehmend stärker werdende ökonomische Interdependenz, eine Vertiefung der neoliberalen Wirtschaftslogik sowie nicht zuletzt die Bildung einer Mitte-rechts-Regierung im Jahr 200o. Diese geänderten Rahmenbedingungen führten zu Zäsuren des Austrokorporatismus und einem Wandel ehemals zentraler SozialpartnerschaftsInstitutionen (z.B. Paritätische Kommission). Die aktuelle Ausprägung der Sozialpartnerschaft unterscheidet sich daher von den Hochblütejahren der späteren 1970er und frühen 1980er Jahre. Angesichts dieser Entwicklungen schien ein nachhaltiger Macht- und Bedeutungsverlust der Sozialpartnerschaft - gerade der Einfluss auf arbeitspolitische Politikbereiche - zu Beginn der 20ooer Jahre mehr als plausibel.

Trotz dieser Kontexte sind das institutionelle Setting der Sozialpartnerschaft und die stabile und privilegierte Einfluss- und Beteiligungsmöglichkeit der Akteure in arbeits- und sozialpolitischen Fragen im internationalen Vergleich in Österreich noch immer einzigartig und von einer hohen Stabilität gekennzeichnet. Es hat gerade in den Zeiten internationaler ökonomischer Krisen als nationaler Modus industrieller Beziehungen eine gute Performance gezeigt (etwa Flecker/Hermann 2015) - so auch während der letzten Banken- und Finanzkrise. In diesem Zusammenhang ist etwa die Aufrechterhaltung der hohen Dichte des Kollektivvertragssystems zu nennen (Pernicka/Stadler 2015) sowie die Funktion des Austrokorporatismus als Legitimationstool für unpopuläre (Austeritäts-) Maßnahmen der Regierung. ${ }^{11}$ So konnte sich die Sozialpartnerschaft vor allem mit der Durchführung der Kurzarbeitsmaßnahmen das Vertrauen der Bevölkerung als verlässlicher politischer und ökonomischer Partner sichern (vgl. Hinterseer 2014).

Seit der Neu-Auflage der Großen Koalition im Jahr 2007 kam es zu einer Reihe von beschlossenen Gesetzen mit sozialpartnerschaftlicher Einbindung, etwa das Anti-Lohn- und Sozialdumpinggesetz und seine Novellierung sowie die Mindestsicherung und die Steuerreform (siehe hierfür auch Krings 2013), um einige zu nennen. Somit kann festgehalten werden,

11 Hier ist auch das im österreichischen, wohlfahrtsstaatlichen Kontext relevante „blame avoidance“ zu berücksichtigen (Fink/Tálos 2004). dass es entgegen politikwissenschaftlicher Prognosen zu Beginn des Millenniums nicht zu einem Ende der Sozialpartnerschaft, sondern sogar zu einem Revival der Einfluss- und Machtspielräume bis hin zu einer Re-Vitalisierung des Austrokorporatismus gekommen ist. Ausgeblieben ist bisher, genau diese Zäsur- und Übergangsphase zu untersuchen, um die daraus abzuleitenden Gründe für die Kontinuität und Stabilität der Sozialpartnerschaft aufzuspüren. Diese Lücke möchte das Papier schließen. Diese Schlüsse sind gerade angesichts der aktuellen und bevorstehenden innenpolitischen Lage für die Einschätzung der zukünftigen Entwicklung der Sozialpartnerschaft von Bedeutung.

Dafür wurde die Phase der Zurückdrängung und Zäsur zwischen den Jahren 2000 und 2006 anhand der Analyse zweier arbeitspolitischer Gesetzgebungsprozesse genauer untersucht, um daraus die einzigartige Beständigkeit dieses nationalen Modells der Arbeitsbeziehungen zu erklären. Ausgehend vom akteurszentrierten Institutionalismus sowie von der Logik der Angemessenheit wurden die Gesetzgebungsprozesse zur Mitarbeitervorsorge („Abfertigung Neu“) (2002) und die Novelle zum Arbeitszeitgesetz (2007) als Fallstudien analysiert. Beide Gesetzesmaterien wurden in der Übergangszeit zwischen dem konstatierten Bedeutungsverlust der Sozialpartnerschaft und einem erneuten Erstarken beziehungsweise Revival der Sozialpartnerschaft verhandelt und beschlossen. Dafür wurden ein „process tracing“ und qualitative problemzentrierte Interviews mit vorwiegend VertreterInnen der Sozialdemokratischen Partei, der Arbeiterkammer und des Gewerkschaftsbundes, aber auch mit Akteuren der Freiheitlichen Partei, der Volkspartei und der Wirtschaftskammer durchgeführt. Wie in diesem Papier deutlich gemacht wurde, gab es starke institutionelle und akteursbezogene Faktoren, die einen nachhaltigen Macht- und Einflussverlust und somit einen nachhaltigen Bedeutungsverlust nach einer ersten massiven Schwächung der Sozialpartnerschaft Anfang der 20ooer Jahre verhinderten. Die Schlussfolgerungen der politikwissenschaftlichen Literatur, auf die mehrmals Bezug genommen wurde und die von einem Ende der Sozialpartnerschaft ausging, müssen größtenteils relativiert werden. Die strukturellen Änderungen - wie etwa die Bedeutungslosigkeit der in den Hochblütejahren wichtigen sozialpartnerschaftlichen Ausschüsse sowie die strukturellen Änderungen des korporatistischen Settings (Karlhofer 2007, Krings 2013, Traxler 1993) - scheinen die Präferenzen und Identitäten der Akteure innerhalb des Settings der Sozialpartnerschaft 
nicht tiefer gehend verändert zu haben. Die Befragungen machten deutlich, dass der Schock bei den InteressensvertreterInnen der ArbeitnehmerInnen, einer Mitte-rechts-Regierung gegenüberzustehen, mittelfristig nicht zu einem nachhaltigen Umdenkprozess des eigenen Selbstverständnisses geführt hat. Die Wiederaufnahme konsensualer, korporatistischer Zusammenarbeit schien nie in Frage gestanden zu sein. Dies lässt sich durch die aus Sicht der Akteure sinnvolle und vor allem bekannte und einschätzbare Konflikt- und Verhandlungssituation mit dem interessenspolitischen „Gegenüber" erklären. Die Streiks gegen die Pensionsreform waren aus Sicht der ArbeitnehmerInnen-Vertretung „erfolgreich“, doch wurden sie in den Befragungen nie als erstrebenswerter Zustand gewertet. Sowohl die Verhandlungen zur neuen Abfertigungsregelung als auch die Verhandlungen zu den im Jahr 2007 beschlossenen Arbeitszeitflexibilisierungen zeugen trotz der gezeigten temporär veränderten Einbindungsabläufe von keinem Wandel innerhalb der Sozialpartnerschaft. Vielmehr deuten sie eher auf eine konservative Struktur dieses Arrangements hin. Die ausgewerteten Einstellungen der Befragten der Sozialdemokratie zum Thema „Rot-Grün-Koalition" unterstreichen diese Schlussfolgerung eines Konservatismus, der den durch stabile und klare Identitätszuschreibungen geprägten Raum von Erwartungssicherheiten schützen will.

Neokorporatistisch orientierte industrielle Beziehungen beziehungsweise Arbeitsbeziehungen „(...) beruhen vornehmlich auf materiellen Legitimationen“ (Czada 2004, 49), bei denen die Stabilität auf der Zufriedenheit mit den Ergebnissen aller Akteure gründet (Czada 2004). Wie nachhaltig diese Grundvoraussetzung angesichts der sich verfestigenden Stagnation der wirtschaftlichen Entwicklung sowie der von Pernicka und Stadler (2015) aufgezeigten „Problemfelder“ der zukünftigen sozialpartnerschaftlichen Entwicklung - zurückbleibende Lohnabschlüsse, zunehmendes Ungleichgewicht zwischen Kapital und Arbeit, zunehmend heterogene ArbeitnehmerInnenschaft - sind, bleibt abzuwarten. Daneben deuten die innenpolitischen Vorzeichen auf eine noch stärkere Schwächung der beiden Großparteien und auf eine damit einhergehende vorläufige Stärkung der Verbände bei gleichzeitigem Erstarken von Parteien mit einer AntiSozialpartnerschaftshaltung hin. In diesem Kontext gilt es auch die berechtigte Frage zu stellen, ob es sich nur um eine „geliehene Stabilität“ (Flecker/Hermann 2005, Pernicka/Hefler 2015) der Sozialpartnerschaft handelt, die bestimmten temporären politisch-ökonomischen
Rahmenbedingungen geschuldet ist. Für die Bewertung einer möglicherweise bevorstehenden erneuten Zäsur der Sozialpartnerschaft können die hier gemachten Erklärungen aus den Jahren 2000 bis 2006 eine Orientierung bieten; außerdem können die hier im Politikfeld Arbeitspolitik identifizierten Erklärungen für die Stabilität und Kontinuität auf einer akteursbezogenen Ebene auch für die Analyse anderer sozialpartnerschaftlicher Politikfelder herangezogen werden: Es kann zu massiven Veränderungen der Sozialpartnerschaft bei gleichzeitiger institutioneller (Flecker/ Hermann 2010) sowie inhaltlicher Kontinuität (Tálos 2015) kommen. Letztendlich geht es darum, wie stabil - unabhängig von den einzelnen Politik- beziehungsweise Betätigungsfeldern - „altbekannte Handlungsund Beziehungsmuster" (Haipeter 2012, 409) sind und bleiben.

\section{Literatur}

Adam, G. (2008): Austria: Industrial relations developments in Europe 2007, European Observatory of Working Life. Online: http://www.eurofound.europa.eu/observatories/ eurwork/comparative-information/national-contributions/austria/austria-industrial-relations-developmentsin-europe-2007 [22.07.2015].

Afonso, A. (2013): Social Concertation in Times of Austerity. European Integration and the Politics of Labour Market Reforms in Austria and Switzerland. Amsterdam: Amsterdam University Press.

Afonso, A. / March, A. (2010): Coming Together But Staying Apart - Continuity and Change in the Austrian and Swiss Varieties of Capitalism. In: Becker, U. (Hg): The Changing Political Economics of Small West European Countries. Amsterdam: Amsterdam University Press, 99-124.

Afonso, A. / Papadopoulos, Y. (2013): Europeanization or Party Politics? Explaining Government Choice for Corporatist Concertation. Governance, 26 (1), 5-29.

Biernacki, P. / Waldorf, D. (1981): Snowball Sampling-Problems and Techniques of Chain Referral Sampling. Sociological Methods \& Research, 10 (2), 141-163.

Baccaro, L. / Simoni, M. (2008): Policy Concertation in Europe: Understanding Government Choice. Comparative Political Studies, 56 (3), 1323-1348.

Beyme, K. von (1997): Der Gesetzgeber. Der Bundestag als Entscheidungszentrum. Opladen:Westdeutscher Verlag.

Culpepper, P. D. (2002): Powering, puzzling, and 'pacting': the informational logic of negotiated reforms. Journal of European Public Policy, 9 (5), 774-79o.

Czada, R. (2004): Konjunkturen des Korporatismus: Zur Geschichte eines Paradigmenwechsels in der Verbände- 
forschung. In: Streeck, W. (Hg): Politische Vierteljahresschrift, Sonderheft 25, 37-63.

Dachs, H. / Gerlich, P. / Gottweis, H. / Kramer, H. / Lauber, V. / Müller, W. C. / Tálos, E. (Hg) (2006): Politik in Österreich: Das Handbuch. Wien: MANZ'sche Verlags- und Universitätsbuchhandlung.

DiePresse.com (2002a): Abfertigung: Sozialpartner setzten sich bei strittigen Punkten durch. Online: http://diepresse.com/home/wirtschaft/economist/252649/Abfertigung_Sozialpartner-setzten-sich-bei-strittigen-Punkten-durch?from=suche.intern.portal [17.11.2010].

DiePresse.com (2002b): Sozialpartner kämpfen gemeinsam für Abfertigungs-Modell. Online:http://diepresse.com/home/ wirtschaft/economist/252814/Sozialpartner-kaempfengemeinsam-fuer-AbfertigungsModell?from=suche.intern.portal [17.11.2010]

Eichhorst, W. / Weishaupt, T. J. (2013): Mit Neo-Korporatismus durch die Krise? Die Rolle des Sozialen Dialogs in Deutschland, Österreich und der Schweiz. IZA Discussion Paper, Forschungsinstitut zur Zukunft der Arbeit, IZA DP No. 7498.

Ennser-Jedenastik, L. (im Erscheinen): Die personelle Verflechtung zwischen Sozialpartnern und Bundesregierung in Österreich, 1945-2015. Österreichische Zeitschrift für Politikwissenschaft.

Fallend, F. / Heinisch, R. (2015): Collaboration as successful strategy against right-wing populism? The case of the centre-right coalition in Austria, 2000-2007, Democratization, 23 (2), 324-344.

Fink, M. / Tálos, E. (2004): Welfare State Retrenchment in Austria: Ignoring the Logic of Blame Avoidance. Journal of Social and Societal Policy, 3 (1), 1-21.

Flecker, J. / Hermann, C. (2015): Mastering the crisis but not the future: the Austrian model in the financial and economic crisis. In: Lehndorff, S. (Hg) (2015): Divisive integrational - The triumph of failed ideas in Europe - revisited. Brussels: European Trade Union Institute (ETUI), 195-208.

Flecker, J. / Hermann, C. (2005): Geliehene Stabilität. Zur Funktionsfähigkeit des dualen Systems der Arbeitsbeziehungen. In: Karlhofer, F. / Tálos, E. (Hg): Sozialpartnerschaft. Österreichische und Europäische Perspektiven. Wien/Münster: LIT-Verlag, 37-56.

George, A. L. / Bennett, A. (2005): Case Studies and Theory Development in the Social Sciences. Bcsia Studies in International Security. Cambridge: MA.

Gerlich, P. (1992): A Farewell to Corporatism. In: Luther, K. R. / Müller, W. C. (Hg): Politics in Austria: Still a Case of Consociationalism. London: Frank Cass, 132-146.

Gerlich, P. / Grande, E. / Müller, W. C. (1988): Corporatism in crisis: stability and change of social partnership in Austria. Political Studies, 36 (2), 209-223.

Haipeter, T. (2012): Sozialpartnerschaft in und nach der Krise: Entwicklungen und Perspektiven. Industrielle Beziehungen, 19 (4), 387-411.
Heinisch, R. (200o): Coping with Economic Integration: Corporatist Strategies in Germany and Austria in the 1990s. West European Politics, 23 (3), 67-96.

Heinisch, R. (2012): Austrian Social Policy Reform in the Era of Integration and Rising Populism. In: Cohen, G. B. / Ansell, B. W. / Gingrich, J. / Cox, R. H. (Hg): Social Policy in the Smaller European Unions States: Berghahn Books, 131-152.

Hinterseer, T. (2014): Sozialpartnerschaft in Zeiten der Krise: Eine Erweiterung der Szenarien zur Zukunft der Sozialpartnerschaft in Österreich. Wirtschafts- und Sozialpolitische Zeitschrift, 37 (2), 135-147.

Jansen, D. (200o): Der neue Institutionalismus. Antrittsvorlesung an der Deutschen Hochschule für Verwaltungswissenschaften. Speyer.

Karlhofer, F. (2007): Filling the Gap? Korporatismus und neue Akteure in der Politikgestaltung. Österreichische Zeitschrift für Politikwissenschaft, 36 (4), 389-403.

Karlhofer, F. (2005): Verbände: Mitgliederorientierung und strategische Neuausrichtung. In: Karlhofer, F. / Tálos, E. (Hg): Sozialpartnerschaft. Österreichische und Europäische Perspektiven. Wien/Münster: LIT-Verlag, 7-35.

Karlhofer, F. / Tálos, E. (2006): Sozialpartnerschaft am Abstieg. In: Tálos, E. (Hg): Schwarz-Blau. Eine Bilanz des „Neu-Regieren“. Wien: LIT-Verlag, 102-116.

Karlhofer, F. / Tálos, E. (200o): Sozialpartnerschaft unter Druck. Trends und Szenarien. In: Pelinka, A. / Plasser, F. / Meixner, W. (Hg): Die Zukunft der österreichischen Demokratie. Trends, Prognosen und Szenarien, 381-402.

Katzenstein, P. J. (1984): Corporatism and Change - Austria, Switzerland, and the Politics of Industry. London: Cornell University Press.

Kittel, B. (200o): Deaustrification? The policy-area-specific evolution of Austrian Social Partnership. West European Politics, 23 (1), 108.

Krings, T. (2013): Von der „Ausländerbeschäftigung“ zur RotWeiß-Rot-Karte: Sozialpartnerschaft und Migrationspolitik in Österreich. Österreichische Zeitschrift für Politikwissenschaft, 42(3), 263-278.

Leibrecht, M. / Rocha-Akis, S. (2014): Sozialpartnerschaft und makroökonomische Performance. WIFO-Monatsberichte, 87 (8), 555-567.

Luther, K. R. / Müller, W. C. (1992): Politics in Austria: Still a Case of Consociationalism. London: Frank Cass.

March, J. G. / Olsen, J. P. (2009): The logic of appropriateness. ARENA Working Papers, 04/09, , Oslo: University of Oslo.

March, J. G. / Olsen, J. P. (1998): The Institutional Dynamics of International Political Orders. International Organization, 52 (4), 943-969.

Mayntz, R. / Scharpf, F. W. (1995). Der Ansatz des akteurzentrierten Institutionalismus. In: Mayntz, R. / Scharpf, F. W. / Max-Planck-Institut für Gesellschaftsforschung (Hg): Gesellschaftliche Selbstregelung und politische Steuerung. Frankfurt a. M.: Campus Verlag, 39-72. 
Nationalrat (2002): 104. Plenarsitzung, XXXI. Gesetzgebungsperiode, 22.05.2002.

Nationalrat (2007a): 9. Plenarsitzung, XXIII. Gesetzgebungsperiode, 16. 01.2007 .

Nationalrat (2007b): Stenografische Protokolle, XXIII. Gesetzgebungsperiode, 16./17.01.2007.

Nationalrat (2001): 77. Plenarsitzung, XXI. Gesetzgebungsperiode, 26.07. 2001.

ÖGB (2002): Stellungnahme zum Gesetzesentwurf zur Implementierung der Betrieblichen Mitarbeitervorsorgekassen.

ÖGB/LWK/AK/WKÖ (2007): Stellungnahme zum Bundesgesetz, mit dem das Arbeitszeitgesetz, das Arbeitsruhegesetz und das Landarbeitsgesetz 1984 geändert werden.

Paster, T. (2014): Why Did Austrian Business Oppose Welfare Cuts? How the Organization of Interests Shapes Business Attitudes Toward Social Partnership. Comparative Political Studies, 47 (7), 966-992.

Pernicka, S. / Hefler. G. (2015): Austrian Corporatism - Erosion or Resilience? Österreichische Zeitschrift für Politikwissenschaft, 44 (3), 39-56.

Perincka, S. / Stadler, B. (2015): Beschäftigungspolitik. In: BEIGEWUM (Hg): Politische Ökonomie Österreichs Kontinuitäten und Veränderungen seit dem EU-Beitritt. Wien: Mandelbaum, 260-292.

Pierson, P. (1996): The new politics of the welfare state. World politics, 48 (2), 143-179.

Reiter, F. (2009): Flexibilität und Sicherheit am Arbeitsmarkt: Flexicurity. Diplomarbeit, Universität Wien.

Scharpf, F. W. (2000): Interaktionsformen - Akteurszentrierter Instiutionalismus in der Politikforschung. Opladen: Leske und Budrich.

Sweeney, J. / Weidenholzer, J. (Hg) (1998): Austria: A Study in Modern Achievement. Avebury: Aldershot.

Tálos, E. (2015): Austrokorporatismus zwischen Kontinuität und Veränderung - EU-Beitritt als ein Bestimmungsfaktor für Veränderungen. In: BEIGEWUM (Hg): Politische Ökonomie Österreichs - Kontinuitäten und Veränderungen seit dem EU-Beitritt. Wien: Mandelbaum, 176-197.

Tálos, E. (2008): Sozialpartnerschaft. Ein zentraler politischer Gestaltungsfaktor in der Zweiten Republik. Wien: Studien Verlag.

Tálos, E. (2006): Sozialpartnerschaft: Austrokorporatismus am Ende? In: Dachs, H. / Gerlich, P. / Gottweis, H. / Kramer, H. / Lauber, V. / Müller, W.C. / Tálos, E. (Hg): Politik in Österreich. Das Handbuch. Wien: MANZ'sche Verlags- und Universitätsbuchhandlung, 425-444.

Tálos, E. (2005): Vom Vorzeige- zum Auslaufmodell? Österreichs Sozialpartnerschaft 1945 bis 2005. In: Karlhofer, F. / Tálos, E. (Hg): Sozialpartnerschaft. Österreichische und Europäische Perspektiven. Wien/Münster: LIT-Verlag, 185-216.

Tálos, E. (2001): Ende der Sozialpartnerschaft? Zäsuren in österreichischer Interessenpolitik. In: Karlhofer, F. / Melchior, J. / Sickinger. H. (Hg): Anlassfall Österreich. Die
EU auf dem Weg zur Wertegemeinschaft. Baden-Baden: Nomos-Verlag, 35-45.

Tálos, E. / Kittel, B. (2001): Gesetzgebung in Österreich: Netzwerke, Akteure und Interaktionen in politischen Entscheidungsprozessen. Wien: WUV-Universitäts-Verlag.

Tálos, E. / Stromberger, C. (2005): Zäsuren in der österreichischen Verhandlungsdemokratie. In: Karlhofer, F. / Tálos, E. (Hg): Sozialpartnerschaft. Österreichische und Europäische Perspektiven. Wien/Münster: LIT-Verlag, 79-108.

Tálos, E. / Stromberger, C. (2004): Verhandlungsdemokratische Willensbildung und korporatistische Entscheidungsfindung am Ende? Einschneidende Veränderungen am Beispiel der Gestaltung des österreichischen Arbeitsrechtes. Österreichische Zeitschrift für Politikwissenschaft, 33 (2), 157-174.

Torpe, L. (2014): Corporatism and Social Trust: Bringing Voluntary Organizations 'Back In'. Journal of Civil Society, 10 (2), 204-218.

Traxler, F. (1996): Sozialpartnerschaft am Scheideweg: Zwischen korporatistischer Kontinuität und neoliberalem Umbruch. Wirtschaft und Gesellschaft, 22 (1), 13-33.

Traxler, F. (1993): Vom Nachfrage- zum Angebotskorporatismus. In: Tálos, E.: Sozialpartnerschaft - Kontinuität und Wandel eines Modells. Wien: Verlag für Gesellschaftskritik, 103-106.

Wineroither, D. M. (2013). Die Sozialpartnerschaft als Eckpfeiler der österreichischen Konsensdemokratie. In: Pellar, B. (Red.): Wissenschaft über Gewerkschaften. Analysen und Perspektiven. Wien, 39-70.

WKÖ (2002): Stellungnahme zum Bundesgesetzentwurf für die Einführung eines neuen Abfertigungsrechts. 


\section{Anhang 1: Übersicht der InterviewpartnerInnen}

\begin{tabular}{|c|c|c|}
\hline Interview & Zeit & Position \\
\hline 1 & September 2012 & Sozialdemokratische Partei Österreichs, Nationalrat, Arbeiterkammer \\
\hline 2 & Oktober 2012 & Sozialdemokratische Partei Österreichs, Klubsekretariat Arbeit und Soziales, Klubdirektion \\
\hline 3 & Dezember 2012 & Arbeiterkammer Wien, Referat Arbeitsmarkt \\
\hline 4 & Dezember 2012 & Arbeiterkammer Wien, Referat Sozialpolitik \\
\hline 5 & Jänner 2013 & Sozialdemokratische Partei Österreichs, Nationalrat, leitende Funktion in einer Teilgewerkschaft des ÖGB \\
\hline 6 & März 2013 & $\begin{array}{l}\text { Sozialdemokratische Partei Österreichs, Nationalrat, zentrale Partei-, Parlaments- und } \\
\text { Gewerkschaftsfunktionen, Ministerin }\end{array}$ \\
\hline 7 & April 2013 & Sozialdemokratische Partei Österreichs, Nationalrat, zentrale Gewerkschaftsfunktionen \\
\hline 8 & April 2013 & Sozialdemokratische Partei Österreichs, Minister \\
\hline 9 & Mai 2013 & Sektionschef Bundesministerium für Arbeit, Soziales und Konsumentenschutz \\
\hline 10 & April 2014 * & Freiheitliche Partei, Minister 2000-2005 \\
\hline 11 & Juni 2014 ** & Wirtschaftskammer, zentrale Funktion \\
\hline 12 & Juli 2014 ** & Österreichische Volkspartei, Bundesminister \\
\hline
\end{tabular}

* Telefoninterview

${ }^{* *}$ Interviewfragen per Mail beantwortet 